\title{
Spin-wave excitations and low-temperature magnetization in the dilute magnetic semiconductor (Ga,Mn)As
}

\author{
Matthias Sperl, ${ }^{1 *}$ Avinash Singh, ${ }^{2}$ Ursula Wurstbauer, ${ }^{1}$ Subrat Kumar Das, ${ }^{2}$ Anand Sharma, ${ }^{3}$ Michael Hirmer, ${ }^{1}$ \\ Wolfgang Nolting, ${ }^{3}$ Christian H. Back, ${ }^{1}$ Werner Wegscheider, ${ }^{1}$ and \\ Günther Bayreuther ${ }^{1}$ \\ ${ }^{1}$ Institut für Experimentelle und Angewandte Physik, Universität Regensburg, 93040 Regensburg, Germany \\ ${ }^{2}$ Department of Physics, Indian Institute of Technology, Kanpur 208016, India \\ ${ }^{3}$ Institut für Physik, Humboldt-Universität zu Berlin, D-12489 Berlin, Germany
}

(Received 13 May 2007; revised manuscript received 20 September 2007; published 14 March 2008)

\begin{abstract}
The spontaneous magnetization as a function of temperature has been investigated for a number of (Ga,Mn)As layers grown on GaAs (001) using superconduction quantum interference device magnetometry. The measurements have been performed with a focus on the low-temperature behavior of the magnetization. The temperature dependence of the spontaneous magnetization $M_{S}$ for $T=0.5 T_{C}$ is found to be well described by Bloch's $T^{3 / 2}$ law for all measured samples. However, we observe an extraordinary enhancement of the spin-wave parameter compared to conventional $3 d$ ferromagnets such as $\mathrm{Fe}$, which implies an enhanced low-temperature decay of the magnetic order. For a thorough understanding of this result, we have theoretically investigated the effect of dilution (disorder) on the finite-temperature spin dynamics and on the spin-wave parameter. It is shown that, due to the preferential accumulation of holes in spin clusters, which weakens the majority bulk spin coupling and results in low-energy magnon softening, there is a strong enhancement in the low-temperature decay of the magnetization with dilution. We find excellent agreement between the calculated and measured values for the spin-wave parameter.
\end{abstract}

DOI: 10.1103/PhysRevB.77.125212

PACS number(s): 75.50.Pp

Diluted magnetic semiconductors (DMSs) based on III-V semiconductors doped with Mn have attracted a lot of interest over the last years motivated by potential applications for these materials in "spintronic" semiconductor devices. ${ }^{1-3}$ The aim is to make use of both the spin and the charge degree of freedom to implement electronic devices ranging from fast nonvolatile memories to quantum computers. ${ }^{3}$ In particular, the discovery of ferromagnetism in $\mathrm{Ga}_{(1-x)} \mathrm{Mn}_{x} \mathrm{As}$, some years ago, in GaAs doped with $\mathrm{Mn}(x=0.01, \ldots, 0.1)$ (Ref. 4) with Curie temperatures up to $T_{C}=173 \mathrm{~K},{ }^{5}$ attracted a lot of interest. Because of the compatibility with the well characterized GaAs system and its relatively high Curie temperature, $(\mathrm{Ga}, \mathrm{Mn}) \mathrm{As}$ is a valuable model system to study the magnetic and electric properties of DMS.

It is widely accepted that substituting $\mathrm{Ga}$ atoms by $\mathrm{Mn}$ in GaAs has a double effect: Mn provides local spin-5/2 magnetic moments, and it acts as an acceptor which supplies holes which, in turn, mediate the exchange coupling between the $\mathrm{Mn}^{2+}$ ions. ${ }^{6}$ Generally, it is accepted that ferromagnetic order in these materials is directly related to the net dopant concentration $x$ and the hole concentration $p$.

$(\mathrm{Ga}, \mathrm{Mn}) \mathrm{As}$ is one of the most extensively studied DMS. However, its temperature dependent magnetization has so far been primarily studied in experiments with respect to the Curie point. ${ }^{7-10}$ In contrast, a few theoretical investigations elucidate the temperature dependence of the magnetization away from the Curie temperature. ${ }^{11,12}$ A detailed experimental and theoretical investigation of the behavior of lowtemperature excitations may give insight in the nature of magnetic excitations in this material and the role of disorder in GaMnAs. In conventional ferromagnets, it has been demonstrated that the temperature dependence of the spontaneous magnetization $M_{S}(T)$ far below the Curie temperature is dominated by long-wavelength spin-wave excitations. The excitation energy $E(k)$ of spin waves in the limit of small wave vectors $(k a \ll 1, a$ is the interatomic distance) is given by

$$
E(k)=\hbar \omega(k)=\Delta+D k^{2} \cdots,
$$

where $\Delta$ is an effective gap due to the dipole-dipole interactions, magnetic anisotropy, or external field. ${ }^{14} D=\mathcal{J} z S a^{2} / 3$ is the spin-wave stiffness coefficient, with $\mathcal{J}$ describing the exchange coupling energy, $S$ the spin quantum number, and $z$ the number of nearest neighbors. The decrease of the spontaneous magnetization $M_{S}$ from its ground state value $M(0)$ is determined by

$$
\frac{M_{S}(0)-M_{S}(T)}{M_{S}(0)}=\frac{\sum n_{k}}{N S} .
$$

Here, $N$ is the number of atoms. $\sum n_{k}$ is the number of excited spin waves and can be obtained as

$$
\sum n_{k}=\int_{1 . B Z} g(\omega)\langle n(\omega)\rangle d \omega,
$$

where $g(\omega)$ is the density of spin-wave states and $\langle n(\omega)\rangle$ the Bose-Einstein statistical law. In the case of a continuous distribution of spin-wave states as in the bulk, Eq. (2) leads to the so-called Bloch's $T^{3 / 2}$ law, ${ }^{15}$

$$
M_{S}(T)=M_{0}\left(1-B T^{3 / 2}\right),
$$

which, originally derived for bulk ferromagnets, is experimentally found to describe very well ultrathin ferromagnetic films in many cases. ${ }^{16-19}$ In conventional spin-wave theory, ${ }^{20}$ 
the spin-wave parameter $B$ and the spin-wave stiffness coefficient $D$ are related by the equation

$$
B=2.612 \frac{g \mu_{B}}{M_{0}}\left(\frac{k_{B}}{4 \pi D}\right)^{3 / 2} .
$$

Here, we report the results of our study of the temperature dependence of magnetization in a DMS well below the Curie temperature. In particular, we investigate whether the decay of the magnetization as a function of temperature behaves similarly to conventional ferromagnetic materials. We find an enhanced decay which may be linked to disorder in the system as seen below.

Sample growth. To substitutionally incorporate $\mathrm{Mn}$ in GaAs, the molecular beam epitaxy (MBE) growth has to be performed at low temperatures. Due to the special growth conditions, the material shows low-temperature (LT)-GaAs defects during the deposition, ${ }^{7}$ such as As antisites and Mn insterstitials, which reduce the concentration of carriers and compensate magnetic moments. Postgrowth annealing of the samples was shown to reduce the number of lattice and positional defects and increase the hole concentration which is accompanied by an increase of $T_{C}$. Because key magnetic properties such as magnetization and Curie temperatures were found to depend very sensitively on compensating defects, these parameters can be improved by an "optimal" postgrowth annealing process. ${ }^{7-9,13}$

The ferromagnetic $(\mathrm{Ga}, \mathrm{Mn})$ As samples discussed in this work have been grown by MBE using a modified Veeco Gen II system. All samples were produced at low-temperature growth conditions on semi-insulating (001) orientated GaAs substrates. After heating of the epiready substrates to $600{ }^{\circ} \mathrm{C}$ in UHV to remove water and oxide from the surface, a $50 \mathrm{~nm}$ thick high-temperature (HT)-AlGaAs buffer layer was deposited at this temperature. Subsequently, an $8 \mathrm{~nm}$ thick LT-GaAs buffer layer was grown $\left(T \approx 250{ }^{\circ} \mathrm{C}\right)$. Finally, $50 \mathrm{~nm}$ thick $\mathrm{Ga}_{1-x} \mathrm{Mn}_{x} \mathrm{As}$ layers were deposited with Mn concentrations of $x=0.03, \ldots, 0.06$. The growth rate for the low-temperature growth was about $0.6 \AA / \mathrm{s}$ ( $T$ $=230-250{ }^{\circ} \mathrm{C}$ ). To ensure an effective postgrowth annealing to remove Mn interstitials, a thick amorphous As layer was deposited on top. Another series of $\mathrm{Ga}_{1-x} \mathrm{Mn}_{x} \mathrm{As}$ samples were grown on a standard $300 \mathrm{~nm}$ HT-GaAs buffer followed by a $10 \mathrm{~nm}$ LT-GaAs layer. Here, the Mn content of the $\mathrm{Ga}_{1-x} \mathrm{Mn}_{x}$ As layer was kept constant at $x=0.06$ and different thicknesses of 100 and $200 \mathrm{~nm}$ were grown also capped with a thick As layer. For details of the growth conditions, see Ref. 21.

Measurements. The magnetic properties of the samples were studied with a superconduction quantum interference device (SQUID) magnetometer over a wide temperature range of $2 \mathrm{~K} \leqslant T \leqslant 180 \mathrm{~K}$. The magnetic anisotropy of the measured $(\mathrm{Ga}, \mathrm{Mn})$ As layers consists of a uniaxial and a fourfold in-plane anisotropy with an easy axis along the [100] direction (Fig. 1). To extract the Curie temperature $T_{C}$, the temperature dependent magnetization was measured during cooling the sample in a field of $10 \mathrm{mT}$ [field cooling (FC)] (Fig. 2). The temperature independent diamagnetic contribution obtained at high fields was subtracted from the hysteresis loops as well as from the $m(T)$ curves.

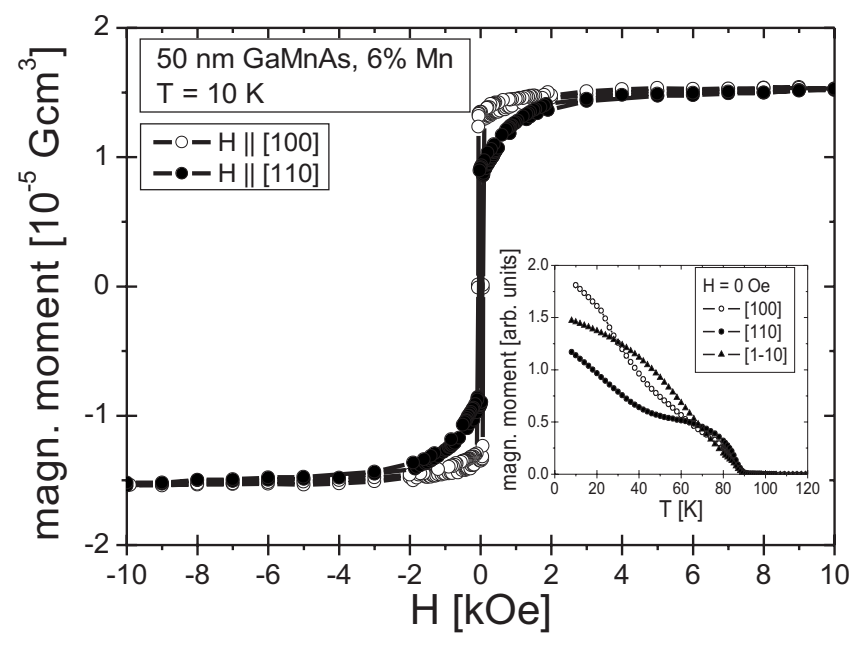

FIG. 1. Field dependence of the magnetization for a $50 \mathrm{~nm}$ $\mathrm{Ga}_{1-x} \mathrm{Mn}_{x}$ As layer with $x=0.06$ (as grown) at $10 \mathrm{~K}$. Two orientations of the film with respect to the magnetic field are shown. Full and empty symbols denote the data taken for the magnetic field along the [110] and [100] crystal directions, respectively. Inset: temperature dependence of the remanent magnetization as measured along three crystal directions. Here, the sample was cooled down through $T_{C}$ in a applied field. Then, at $10 \mathrm{~K}$, the field is removed and the remanent magnetization is measured. In this case, remanence represents the projection of the magnetization along the crystal direction. A temperature-driven reorientation transition can be observed.

The Curie temperature was taken as the inflection point of the measured $m(T)$ curve. Figure 2 shows the results for these measurements for a $50 \mathrm{~nm}$ thick $\mathrm{Ga}_{1-x} \mathrm{Mn}_{x}$ As sample with $x=0.06$ as grown as well as after postgrowth annealing of the same sample. In dependence on Myers et al., ${ }^{22}$ hole density data are taken from Hall measurements in magnetic fields up to $\pm 0.7 \mathrm{~T}$ at room temperature and in addition to that in magnetic fields up to $\pm 13 \mathrm{~T}$ at $T=4.2 \mathrm{~K}$ from samples patterned in a standard Hall geometry.

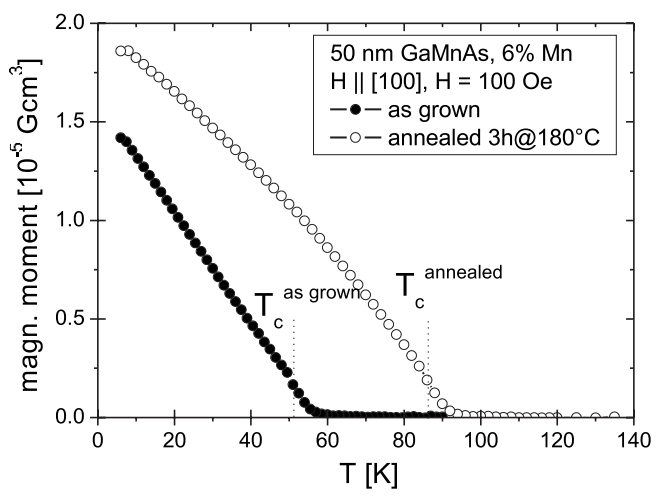

FIG. 2. Temperature dependence of FC magnetic moment in an applied field $\mu_{0} H=10 \mathrm{mT}$ for a $50 \mathrm{~nm} \mathrm{Ga}{ }_{1-x} \mathrm{Mn}_{x}$ As layer on GaAs (001) (with AlGaAs buffer layer) and $x=0.06$. The field was applied along the GaAs [100] direction. The solid symbols are measurements for the as grown sample; the open symbols represent the same sample after annealing for $3 \mathrm{~h}$ at $180^{\circ} \mathrm{C}$ in air. 


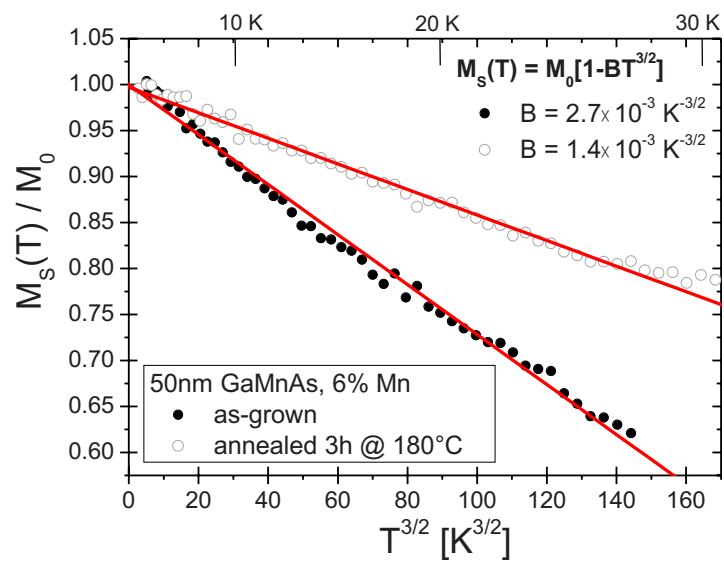

FIG. 3. (Color online) Temperature dependence of the spontaneous magnetization $M_{S}(T)$ normalized to $M_{0}$ for a $50 \mathrm{~nm}$ $\mathrm{Ga}_{1-x} \mathrm{Mn}_{x}$ As layer on GaAs (001) (with AlGaAs buffer layer) and $x=0.06$. The field was applied along the GaAs [100] direction. The solid symbols are measurements for the as-grown sample; the open symbols represent the same sample after annealing for $3 \mathrm{~h}$ at $180{ }^{\circ} \mathrm{C}$ in air. The solid lines represent a fit according to Bloch's $T^{3 / 2}$ law.

To allow a quantitative description of the decay of the magnetization, we measured the temperature dependence of the spontaneous magnetization $M_{S}(T)$ for all layers in the following way. The spontaneous magnetic moment $m_{S}(T)$ of the GaMnAs film is determined by a linear extrapolation of the measured $m(H)$ curve along the [100] direction between 7 and $4.8 \mathrm{kOe}$ to zero for all temperatures. The field was chosen to be high enough to completely align the magnetization parallel to the external field. This method has the additional advantage that temperature-driven reorientation transitions that are observed in GaMnAs (as can be seen in the inset of Fig. 1) have no influence on the determination of the spontaneous magnetization $M_{S}(T)$. Figure 3 shows the data obtained in this way for the $50 \mathrm{~nm} \mathrm{Ga} \mathrm{Ga}_{1-x} \mathrm{Mn}_{x}$ As layer with $x=0.06$ (as grown and annealed) in the temperature range between 2 and $34 \mathrm{~K}$ normalized to its value at $2 \mathrm{~K}\left(m_{0}\right)$ and plotted versus $T^{3 / 2}$. Due to the extrapolation from high fields to zero and the small ferromagnetic signal compared to the diamagnetic contribution of the GaAs substrate, the measurements show a relatively large noise. However, it can be seen that the temperature dependence of magnetization can be well described by Bloch's $T^{3 / 2}$ law and one can derive the spin-wave parameter $B$ from the measurements. Table I gives a summary of the spin-wave parameters for different thicknesses and $\mathrm{Mn}$ contents of the (Ga,Mn)As layer.

From the data of Fig. 3, it can be seen that after annealing, the spin-wave parameters $B$ are reduced by about $50 \%$ compared to the as-grown sample. This behavior can be understood if we take into account the role of the exchange interaction for spin-wave excitations. According to Eq. (5), the spin-wave parameter $B$ is associated with the spin-wave stiffness coefficient $D$ which is, in turn, directly proportional to the exchange integral $\mathcal{J}$. On the other hand, in the molecularfield approximation, the exchange coupling constant $\mathcal{J}$ between nearest neighbors is proportional to the Curie temperature $T_{C}$ by ${ }^{23,24,39}$

$$
T_{C}=\left[2 S(S+1) / 3 k_{B}\right] z \mathcal{J} .
$$

Experimentally, it is well known that for Mn concentrations above 2\%-3\%, Mn atoms can occupy interstitial sites and act as donors, which reduce the hole concentration and the total magnetic moment by antiferromagnetic coupling to substitutional $\mathrm{Mn} .{ }^{25}$ Postgrowth annealing at temperatures at or below the growth temperature reduces the number of $\mathrm{Mn}$ interstitials in the GaMnAs layer. ${ }^{13}$ Indeed, a corresponding increase of the magnetization $M$ and the hole concentration $p$ is observed. According to theoretical predictions, ${ }^{26}$ a larger hole concentration leads to an increase of the average exchange constant $\mathcal{J}$ which, in turn, raises $T_{C}$. The present experiment confirms this interpretation as an increase of $T_{C}$ and a reduced spin-wave parameter $B$ is observed after annealing, the later being proportional to $\mathcal{J}^{-3 / 2}$.

The related spin-wave stiffness coefficient values $D$, listed in Table I, are in good agreement with results from ferromagnetic resonance measurements in as-grown (Ga,Mn)As samples reported in Ref. 27 , where $D \approx 60 \mathrm{meV} \AA^{2}$ was

TABLE I. Ground state magnetization $M(0)$, Curie temperature $T_{C}$, and spin-wave parameter $B$ as extracted from the SQUID measurements for $(\mathrm{Ga}, \mathrm{Mn})$ As samples with different thicknesses, Mn content and postgrowth annealing. The spin-wave stiffness coefficient was calculated using Eq. (5) and the experimentally determined values of $B$. For comparison the results for a $200 \mathrm{~nm}$ thick (bulk) Fe layer grown epitaxial on GaAs (001) are also given. The hole concentrations $p$ are taken from Hall measurements in a standard Hall geometry at room temperature and $4.2 \mathrm{~K}$.

\begin{tabular}{|c|c|c|c|c|c|c|c|c|c|}
\hline $\begin{array}{l}\text { Thickness } \\
(\mathrm{nm})\end{array}$ & $\begin{array}{l}\mathrm{Mn} \\
(\%)\end{array}$ & Annealed & $\begin{array}{l}T_{C} \\
(\mathrm{~K})\end{array}$ & $\begin{array}{c}M(0) \\
(\mathrm{G})\end{array}$ & $\begin{array}{c}B \\
\left(10^{-3} \mathrm{~K}^{-3 / 2}\right)\end{array}$ & $B_{3 / 2}$ & $\begin{array}{c}D \\
\left(\mathrm{meV} \AA^{2}\right)\end{array}$ & $\begin{array}{c}p \\
\left(1 / \mathrm{cm}^{3}\right), T=298 \mathrm{~K}\end{array}$ & $\begin{array}{c}p \\
\left(1 / \mathrm{cm}^{3}\right), T=4.2 \mathrm{~K}\end{array}$ \\
\hline 50 & 6 & No & $51 \pm 4$ & 27.1 & $2.7 \pm 0.2$ & $1.0 \pm 0.1$ & 53 & $3.6 \times 10^{19}$ & $1.3 \times 10^{20}$ \\
\hline 50 & 6 & Yes & $86 \pm 3$ & 34.3 & $1.4 \pm 0.1$ & $1.1 \pm 0.1$ & 70 & $\ldots$ & $2 \times 10^{20}$ \\
\hline 50 & 3 & No & $37 \pm 3$ & 16.5 & $3.2 \pm 0.1$ & $0.7 \pm 0.1$ & 66 & $6.3 \times 10^{19}$ & $1 \times 10^{20}$ \\
\hline 100 & 6 & No & $63 \pm 3$ & 33.3 & $1.5 \pm 0.1$ & $0.8 \pm 0.1$ & 68 & $6.9 \times 10^{19}$ & $\ldots$ \\
\hline 200 & 6 & No & $57 \pm 2$ & 22 & $1.6 \pm 0.1$ & $0.7 \pm 0.1$ & 86 & $6.7 \times 10^{19}$ & $3.2 \times 10^{20}$ \\
\hline 200 & 6 & Yes & $69 \pm 3$ & 24.8 & $1.0 \pm 0.1$ & $0.6 \pm 0.1$ & 109 & $1.5 \times 10^{20}$ & $6.6 \times 10^{20}$ \\
\hline $200 \mathrm{~nm} \mathrm{Fe}$ & $\cdots$ & $\cdots$ & 1043 & 1766 & 0.0052 & 0.18 & 211 & $\cdots$ & $\cdots$ \\
\hline
\end{tabular}

${ }^{\text {aReference } 16 .}$ 
found. The increase of $D$ upon annealing has also been found by Wang et al. ${ }^{28}$ from pump-probe experiments. Recently, the opposite behavior, i.e., a decrease of $D$ during annealing, has been concluded by Zhou et al. ${ }^{29}$ from their measurements of ferromagnetic resonance in $(\mathrm{Ga}, \mathrm{Mn})$ As films, while also an increase of $T_{C}$ was observed. Here, the validity of several assumptions made for the extraction of the spin-wave stiffness parameter $D$ from spin-wave resonance fields will have to be checked in future experiments.

The most prominent feature emerging from the present investigation is the order of magnitude of the spin-wave parameter $B$ for the measured (Ga,Mn)As layers. The determined spin-wave parameter $B(\mathrm{GaMnAs})$ is about 100 times larger than $B(3 d)$ for conventional $3 d$ ferromagnets. To allow a reasonable comparison between the spin-wave parameters $B$ for different ferromagnetic systems (despite the difference in the Curie temperature), one can use the normalized coefficient $B_{3 / 2}=B T_{C}^{3 / 2}$, as defined by an alternative notation of Eq. (4), ${ }^{30}$

$$
\frac{M_{S}(0)-M_{S}(T)}{M_{S}(0)}=B_{3 / 2}\left(T / T_{C}\right)^{3 / 2} .
$$

For this reason, Table I contains also the corresponding values of $B_{3 / 2}$ for GaMnAs and Fe for comparison. It is evident that the reduced coefficients $B_{3 / 2}$ for the measured GaMnAs samples are still roughly three to six times larger than for Fe.

Similar observations have been made from studies on amorphous ferromagnetic alloys in comparison with crystalline ferromagnets ${ }^{23,30}$ where the reduced coefficient $B_{3 / 2}$ for the amorphous alloys is considerably enhanced compared to the crystalline ferromagnets. It has been argued that structural disorder results in a reduction of the exchange coupling and therefore an enhancement of spin excitations. ${ }^{30}$

It has to be noted that within the error bars, only those values of $B_{3 / 2}$ in Table I are in good agreement, which have been measured on the same piece of sample (as grown and annealed). However, among different samples, a deviation in the normalized coefficient $B_{3 / 2}$ can be seen although some of the samples may possess similar Curie temperatures. This fact can be understood considering the different growth conditions of the measured samples that have a large effect on the structural and magnetic properties of the $(\mathrm{Ga}, \mathrm{Mn}) \mathrm{As}$ samples and can cause a different degree of structural disorder. We would like to emphasize that $B_{3 / 2}$ is more sensitive to disorder than the Curie temperature. Details of the influence of disorder will be presented in the next paragraph.

Large enhancement of spin-wave parameter $B$ in a diluted magnet. In order to quantitatively understand the microscopic origin of this large enhancement of the spin-wave parameter $B$ in DMS systems, we have theoretically investigated the effect of dilution on the finite-temperature spin dynamics and on $B$ in a diluted magnet. For this purpose, we consider the minimal model,

$$
H=t \sum_{i, \delta, \sigma} a_{i, \sigma}^{\dagger} a_{i+\delta, \sigma}+\epsilon_{d} \sum_{I, \sigma} a_{I, \sigma}^{\dagger} a_{I, \sigma}-\frac{J}{2} \sum_{I} \mathbf{S}_{I} \cdot \sigma_{I},
$$

used conventionally to study the interplay between itinerant carriers in a partially filled band and localized impurity mo- ments in DMS systems such as $(\mathrm{Ga}, \mathrm{Mn}) \mathrm{As}$. The mixed spinfermion model [Eq. (8)] includes a hopping term representing the host semiconductor valence band and an exchange coupling between the randomly placed $S=5 / 2 \mathrm{Mn}^{++}$impurity spins $S_{I}$ and the fermion spin density $\sigma_{I} / 2$ at impurity sites $I$. The impurity on-site energy $\epsilon_{d}$ represents the energy difference between host and impurity sites due to chemical substitution. By tuning the impurity-level position relative to the host band, the density of states can be made to resemble that obtained from band structure calculations. ${ }^{31}$ For simplicity, we consider Eq. (8) on a simple cubic (sc) lattice with periodic boundary conditions, with $N_{m}$ as magnetic impurities placed randomly on a fraction $x \equiv N_{m} / N$ of the $N=L^{3}$ host sites $i$. In our calculations, for the sc lattice, $4 x$ corresponds to $x$ in the face centered cubic (fcc) lattice of (Ga,Mn)As which has four Ga sites per unit cell and therefore manganese impurity concentration $4 x$ per unit cell. We set $t=1$ as the unit of the energy scale.

Recently, the spectral and spatial features of magnon excitations in the model [Eq. (8)] were examined within an exact treatment of impurity positional disorder and a nonperturbative treatment of the exchange coupling. ${ }^{32}$ The magnon propagator was studied in the random phase approximation (RPA), where the bubble diagrams, representing

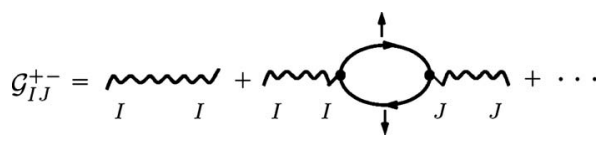

repeated interactions between impurity spins mediated by the particle-hole bubble, provide the lowest-level spinrotationally symmetric treatment of transverse spin fluctuations where the Goldstone mode is explicitly preserved. Finite-temperature spin dynamics was studied using a locally self-consistent magnon renormalization scheme, and the temperature dependence of average magnetization was found to be well described by the form $\left\langle S_{z}\right\rangle=S\left(1-B T^{3 / 2}\right)$ at low temperature. We will examine the dilution $(x)$ dependence of the spin-wave parameter $B$. The above approach is briefly summarized below.

Due to spin-rotation symmetry of Eq. (8), the impurity spin dynamics is naturally described by an isotropic effective interaction $-\Sigma_{I J} \mathcal{J}_{\mathcal{I} \mathcal{J}} \mathbf{S}_{I} \mathbf{S}_{J}$ between impurity spins, and at the RPA level, the (weakly dynamical) carrier-mediated spin couplings are given by

$$
\mathcal{J}_{I J}(\omega)=\frac{J^{2}}{4}\left[\chi^{0}(\omega)\right]_{I J},
$$

in terms of the particle-hole bubble,

$$
\begin{gathered}
{\left[\chi^{0}(\omega)\right]_{I J}=i \int \frac{d \omega}{2 \pi}\left[G^{\uparrow}\left(\omega^{\prime}\right)\right]_{I J}\left[G^{\downarrow}\left(\omega^{\prime}-\omega\right)\right]_{J I}} \\
=\sum_{l, m} \frac{\psi_{l \uparrow}^{J} \psi_{l \uparrow}^{J} \psi_{m \downarrow}^{I} \psi_{m \downarrow}^{J}}{E_{m \downarrow}-E_{l \uparrow}+\omega} f_{l \uparrow}\left(1-f_{m \downarrow}\right) \\
+\sum_{l, m} \frac{\psi_{l \uparrow}^{I} \psi_{l \uparrow}^{J} \psi_{m \downarrow}^{I} \psi_{m \downarrow}^{J}}{E_{l \uparrow}-E_{m \downarrow}-\omega}\left(1-f_{l \uparrow}\right) f_{m \downarrow},
\end{gathered}
$$

evaluated by integrating out the fermions in the broken- 
symmetry state. The spin couplings determine the bare magnon Hamiltonian, with off-diagonal and diagonal matrix elements given by ${ }^{32} \mathcal{H}_{I \neq J}=-2 S \mathcal{J}_{I J}$ and $H_{I I}=\Sigma_{J \neq I} 2 S \mathcal{J}_{I J}$, respectively. As magnon energies are very low compared to the Stoner gap, the $\omega$ dependence of $[\mathcal{H}]$ is sufficiently weak to be neglected, and the eigenvalues and eigenvectors $[\mathcal{H}]$ $\left|\phi_{l}^{0}\right\rangle=\omega_{l}^{0}\left|\phi_{l}^{0}\right\rangle$ directly yield the (bare) magnon energies $\left\{\omega_{l}^{0}\right\}$ and wave functions $\left\{\phi_{l}^{0}\right\}$. Although their nearly static nature is similar to that of the Ruderman-Kittel-Kasuya-Yoshida interaction, the spin couplings are strongly nonpertubative in character due to the $J$ and $x$ dependence of the fermion wave functions and eigenvalues in Eqs. (10a) and (10b).

The above isotropic spin interaction neglects magnetic anisotropy arising from spin-orbit interaction in the host semiconductor, ${ }^{33-36}$ and the recently observed strain-induced uniaxial anisotropy which is dependent on hole concentration and temperature. ${ }^{37}$

To evaluate the spin couplings, we consider the fully polarized, collinear ferromagnetic ground state at $T=0 \mathrm{~K}$, stability of which is confirmed by the absence of negativeenergy magnon modes. The fermion eigenvalues $\left\{E_{l \sigma}\right\}$ and wave functions $\left\{\psi_{l \sigma}\right\}$ are obtained by exact diagonalization of the $N \times N$ fermion Hamiltonian with effective impurity potentials $\epsilon_{d} \mp J S / 2$ for the two fermion spins. Furthermore, we consider the saturated ferromagnetic state with a fully occupied spin- $\uparrow$ band $\left(p \equiv N_{\downarrow}^{\text {holes }} / 2 N\right.$ and hole doping only in the pushed-up spin- $\downarrow$ band), so that only the first term in Eq. (10b) contributes to the particle-hole bubble. For $p \sim x$ or lower values of $J$ and $x$, we do obtain negative-energy magnon modes, indicating noncollinear ferromagnetic ordering, as found in earlier studies. ${ }^{38}$

From the spin couplings, the average magnetization $\left\langle S_{z}\right\rangle$ was evaluated self-consistently using the Callen formula for a quantum spin- $S$ ferromagnet, ${ }^{39}$

$$
\left\langle S_{z}\right\rangle=\frac{(S-\Phi)(1+\Phi)^{2 S+1}+(S+1+\Phi) \Phi^{2 S+1}}{(1+\Phi)^{2 S+1}-\Phi^{2 S+1}},
$$

in terms of the configuration- and site-averaged boson occupation number,

$$
\Phi \equiv\left\langle\Phi_{I}\right\rangle=\left\langle\frac{1}{N_{m}} \sum_{l} \frac{1}{e^{\beta \omega_{l}}-1}\right\rangle_{c},
$$

where the magnon energies $\omega_{l} \equiv\left(\left\langle S_{z}\right\rangle / S\right) \omega_{l}^{0}$ are uniformly renormalized as in the standard Tyablikov theory. Configuration and site averaging are particularly convenient in this globally self-consistent magnon-renormalization scheme, and at low temperatures where $\left\langle S_{I}^{z}\right\rangle \sim S$, it has been shown to be asymptotically degenerate with a locally self-consistent renormalization scheme. ${ }^{32}$

In the following, we consider a realistic carrier bandwidth $W=12 t=10 \mathrm{eV}$ of the order of that for the host semiconductor GaAs. Also, for $J / t$ in the range of $2-4$, the exchange coupling $J \sim W / 4 \sim 2.5 \mathrm{eV}$ is in the experimental range, as direct measurements yield $J=1.2 \pm 0.2 \mathrm{eV}$ from core-level photoemission ${ }^{40}$ and $J=2.4 \pm 0.9 \mathrm{eV}$ from magnetotransport. ${ }^{41,42}$ The combination of $J=2$ and $\epsilon_{d}=2$ shifts the impurity states near the band edge, producing a

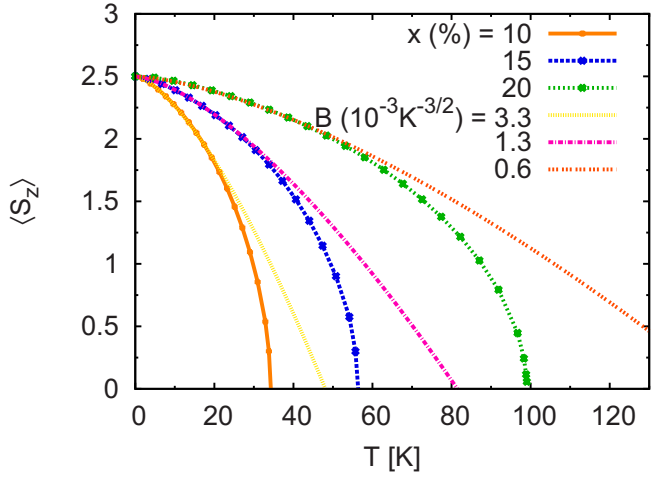

FIG. 4. (Color online) Configuration-averaged magnetization for a $N=10^{3}$ system at three different dilutions along with fits with the Bloch form $S\left(1-B T^{3 / 2}\right)$ for carrier bandwidth $W=12 t=10 \mathrm{eV}, p / x$ $=1 / 10, J=4$, and $\epsilon_{d}=0$.

peak (resonance), ${ }^{31}$ as in density-functional calculations, ${ }^{43}$ where the minority-spin impurity-band peak is above the Fermi energy and strongly locally hybridized.

Figure 4 illustrates the temperature dependence of configuration-averaged magnetization evaluated from Eqs. (11) and (12) for three different dilutions and a fixed fractional hole concentration $p / x=1 / 10$. As seen from Fig. 4 , the low-temperature behavior of magnetization fits well with the Bloch form $S\left(1-B T^{3 / 2}\right)$ for all considered cases, which allows the spin-wave parameter to be extracted for different dilutions $x$. Figure 5 shows the behavior of $B$ with dilution $x$, the magnitude of $B$ changing from $\leq 0.1 \times 10^{-3} \mathrm{~K}^{-3 / 2}$ in the concentrated $(x \sim 1)$ limit to $\sim 5 \times 10^{-3} \mathrm{~K}^{-3 / 2}$ in the diluted $(x \sim 1 / 10)$ limit. The drastic enhancement of nearly 2 orders of magnitude of $B$ with dilution, with $B$ of the same order of magnitude in the dilution limit as obtained in SQUID measurements of $(\mathrm{Ga}, \mathrm{Mn}) \mathrm{As}$, provides a microscopic understanding of the finite-temperature spin dynamics in diluted magnets and also of the large observed ratio $B(\mathrm{GaMnAs}) / B(3 d)$.

Turning to the magnetic behavior near $T_{C}$ and the role of disorder on the reduced coefficient $B_{3 / 2}=B T_{C}^{3 / 2}$, we compare the magnetization behavior for the disordered and ordered cases (Fig. 6), calculated using the locally self-consistent

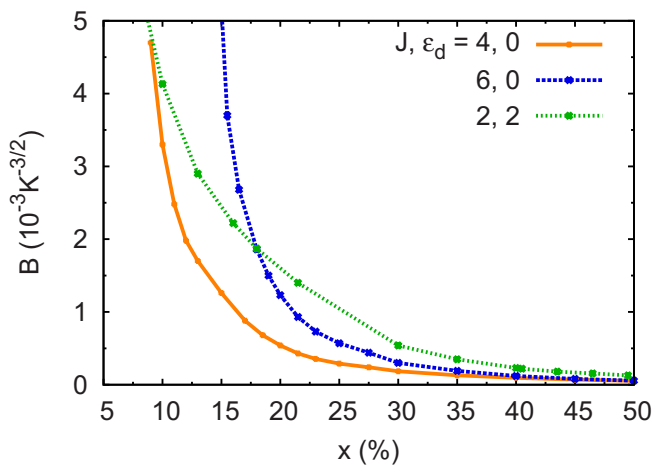

FIG. 5. (Color online) Variation of $B$ with impurity concentration $x$ for carrier bandwidth $W=12 t=10 \mathrm{eV}, p / x=1 / 10$, and $\left(J, \epsilon_{d}\right)=(4,0),(6,0)$, and $(2,2)$. 


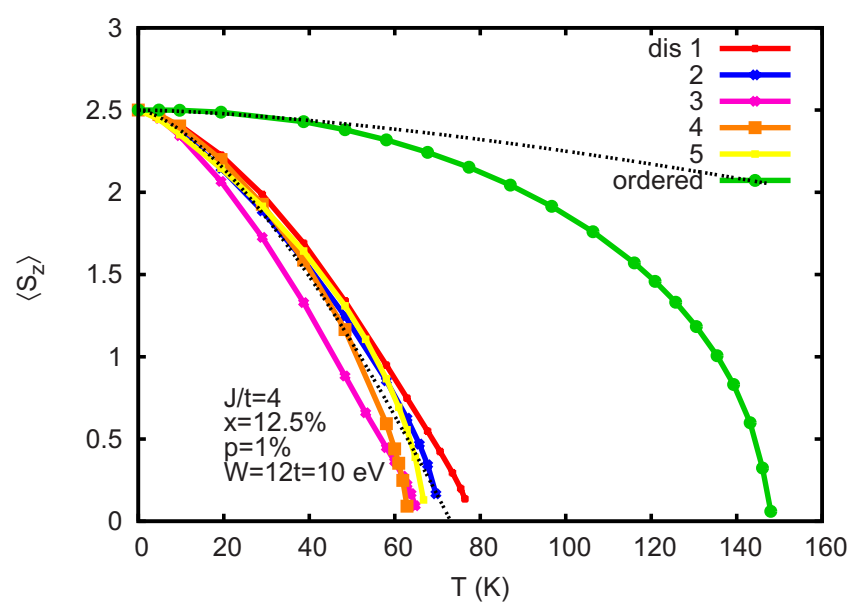

FIG. 6. (Color online) Site-averaged magnetization shown for five disorder configurations of a $N=12^{3}$ system in comparison with the ordered case for exactly the same dilution $x=1 / 8$. Also shown (dashed lines) are fits with the Bloch form $S\left(1-B T^{3 / 2}\right)$.

scheme which incorporates local variations in the magnon occupation number $\Phi_{I}$ and magnetization $\left\langle S_{I}^{z}\right\rangle$. The distinctly slower magnetization decay near $T_{C}$ for the disordered cases is due to strong cluster couplings and correlations which tend to prolong magnetic order near $T_{C}$. As the slower magnetization fall off is consequently closer to the Bloch $T^{3 / 2}$ form in the whole temperature range, Eq. (4) then directly implies $B_{3 / 2}^{d i s} \sim 1$ for the disordered case. On the other hand, the ordered (diluted) case is characterized by a much slower fall off at low temperature $\left(B \approx 10^{-4} \mathrm{~K}^{-3 / 2}\right)$ but a sharp fall off near $T_{C}(\approx 150 \mathrm{~K})$. For this case, we obtain $B_{3 / 2}^{\text {ord }} \sim 0.18$, which is nearly the same as for a typical $3 d$ transition metal case, and also close to the observed value for iron, indicating universal behavior dependent only on the shape of the magnetization curve.

The magnitude of the reduced spin-wave coefficient $B_{3 / 2}$ is seen to provide a composite measure of the two independent effects of disorder in a diluted magnet involving very different energy scales. While the large density of lowenergy magnetic excitations due to weakened bulk spin couplings results in strong enhancement of the spin-wave parameter $B$ with dilution, the strong cluster couplings and correlations prolong the magnetic order near $T_{C}$, bringing the magnetization decay closer to the Bloch $T^{3 / 2}$ form and resulting in $B_{3 / 2}^{\text {dis }} \sim 1$.

In summary, we have shown that the temperature dependence of the magnetization for the measured (Ga,Mn)As layers can be well described using Bloch's law. Interestingly, our investigations have shown an unexpectedly strong thermal decay of the magnetization. This large enhancement of the spin-wave parameter $B$ can be attributed to a large enhancement of the density of low-energy magnetic excitations with dilution. This strong magnon softening has been recently shown to be a consequence of significant weakening of bulk spin couplings, resulting in turn from the preferential accumulation of holes in spin clusters and consequent depletion of holes from the bulk spins which support the lowenergy extended-state magnon modes. ${ }^{44}$ Reducing spin clustering and impurity disorder therefore enhances ferromagnetism blackin the studied doping range.

Furthermore, the determination of the reduced spin-wave coefficient proves to be an efficient diagnostic tool for the degree of disorder of Mn ions in the DMS GaMnAs.

Financial support by the DFG through the SFB 689 is gratefully acknowledged. A. Singh acknowledges support from the Alexander von Humboldt Foundation. *matthias.sperl@physik.uni-regensburg.de

${ }^{1}$ H. Ohno, Science 281, 951 (1998).

${ }^{2}$ Semiconductor Spintronic and Quantum Computation, edited by D. D. Awschalom, N. Samarth, and D. Loss (Springer, Heidelberg, 2002).

${ }^{3}$ I. Zutic, J. Fabian, and S. D. Sarma, Rev. Mod. Phys. 76, 323 (2004).

${ }^{4}$ H. Ohno, A. Shen, F. Matsukura, A. Oiwa, A. Endo, S. Katsumoto, and Y. Iye, Appl. Phys. Lett. 69, 363 (1996); J. De Boeck, R. Oesterholt, H. Bender, A. Van Esch, C. Bruynseraede, C. Van Hoof, and G. Borghs, J. Magn. Magn. Mater. 156, 148 (1996).

${ }^{5}$ K. Y. Wang, R. P. Campion, K. W. Edmonds, M. Sawicki, T. Dietl, C. T. Foxon, and B. L. Gallagher, AIP Conf. Proc. 772, 333 (2005).

${ }^{6}$ H. Ohno, J. Magn. Magn. Mater. 200, 110 (1999).

${ }^{7}$ V. Stanciu, O. Wilhelmsson, U. Bexell, M. Adell, J. Sadowski, J. Kanski, P. Warnicke, and P. Svedlindh, Phys. Rev. B 72, 125324 (2005).

${ }^{8}$ K. W. Edmonds, K. Y. Wang, R. P. Campion, A. C. Neumann, N. R. S. Farley, B. L. Gallagher, and C. T. Foxon, Appl. Phys. Lett. 81, 4991 (2002).
${ }^{9}$ D. Chiba, K. Takamura, F. Matsukura, and H. Ohno, Appl. Phys. Lett. 82, 3020 (2003).

${ }^{10}$ S. J. Potashnik, K. C. Ku, S. H. Chun, J. J. Berry, N. Samarth, and P. Schiffer, Appl. Phys. Lett. 79, 1495 (2001).

${ }^{11}$ T. Jungwirth, J. Masek, K. Y. Wang, K. W. Edmonds, M. Sawicki, M. Polini, Jairo Sinova, A. H. MacDonald, R. P. Campion, L. X. Zhao, N. R. S. Farley, T. K. Johal, G. van der Laan, C. T. Foxon, and B. L. Gallagher, Phys. Rev. B 73, 165205 (2006).

${ }^{12}$ S. Das Sarma, E. H. Hwang, and A. Kaminski, Phys. Rev. B 67, 155201 (2003).

${ }^{13}$ K. W. Edmonds, P. Boguslawski, K. Y. Wang, R. P. Campion, S. N. Novikov, N. R. S. Farley, B. L. Gallagher, C. T. Foxon, M. Sawicki, T. Dietl, M. B. Nardelli, and J. Bernholc, Phys. Rev. Lett. 92, 037201 (2004).

${ }^{14}$ J. D. Axe, G. Shirane, T. Mizoguchi, and K. Yamauchi, Phys. Rev. B 15, 2763 (1977).

${ }^{15}$ F. Bloch, Z. Phys. 61, 206 (1930).

${ }^{16}$ W. Kipferl, M. Sperl, T. Hagler, R. Meier, and G. Bayreuther, J. Appl. Phys. 97, 10B313 (2005).

${ }^{17}$ G. Lugert and G. Bayreuther, Phys. Rev. B 38, 11068 (1988).

${ }^{18}$ M. Stampanoni, A. Vaterlaus, M. Aeschlimann, and F. Meier, 
Phys. Rev. Lett. 59, 2483 (1987).

${ }^{19}$ M. Sperl, W. Kipferl, M. Dumm, and G. Bayreuther, J. Appl. Phys. 99, 08J703 (2006).

${ }^{20}$ For a review of elementary spin-wave theory, see F. Keffer, in Ferromagnetism, edited by H. P. J. Wijn (Spinger-Verlag, Berlin, 1966), Vol. XVIII, Pt. 2, p. 1.

${ }^{21}$ M. Reinwald, U. Wurstbauer, M. Döppe, W. Kipferl, K. Wagenhuber, H.-P. Tranitz, D. Weiss, and W. Wegscheider, J. Cryst. Growth 278, 690 (2005).

${ }^{22}$ R. C. Myers, B. L. Sheu, A. W. Jackson, A. C. Gossard, P. Schiffer, N. Samarth, and D. D. Awschalom, Phys. Rev. B 74, 155203 (2006).

${ }^{23}$ S. N. Kaul and T. V. S. M. Mohan Babu, J. Phys.: Condens. Matter 1, 8509 (1989).

${ }^{24}$ Within a spin-fluctuation theory, the expression for the Curie temperature $T_{C}$, differs from the molecular-field approximation by a "spin-fluctuation factor" $F$. Considering this, one gets the following equation determining the Curie temperature, $T_{C}$ $=\frac{2(S+1)}{9 k_{B} F^{2}(-1)}[(4 S+1) F(-1)-(S+1)] z J$, with $F(-1)=1.51638(\mathrm{sc})$, 1.39320 (bcc), and 1.34466 (fcc).

${ }^{25}$ K. Y. Wang, K. W. Edmonds, R. P. Campion, B. L. Gallagher, N. R. S. Farley, C. T. Foxon, M. Sawicki, P. Boguslawski, and T. Dietl, J. Appl. Phys. 95, 6512 (2004).

${ }^{26}$ T. Dietl, H. Ohno, and F. Matsukura, Phys. Rev. B 63, 195205 (2001).

${ }^{27}$ S. T. B. Goennenwein, T. Graf, T. Wassner, M. S. Brandt, M. Stutzmann, J. B. Philipp, R. Gross, M. Krieger, K. Zürn, P. Ziemann, A. Koeder, S. Frank, W. Schoch, and A. Waag, Appl. Phys. Lett. 82, 730 (2003).

${ }^{28}$ D. M. Wang, Y. H. Ren, X. Liu, J. K. Furdyna, M. Grimsditch, and R. Merlin, Phys. Rev. B 75, 233308 (2007).
${ }^{29}$ Ying-Yuan Zhou, Yong-Jin Cho, Zhiguo Ge, Xinyu Liu, Malgorzata Dobrowolska, and Jacek K. Furdyna, AIP Conf. Proc. 893, 1213 (2007).

${ }^{30}$ S. N. Kaul, Phys. Rev. B 24, 6550 (1981).

${ }^{31}$ R. Bouzerar, G. Bouzerar, and T. Ziman, Europhys. Lett. 78, 67003 (2007).

${ }^{32}$ A. Singh, S. K. Das, A. Sharma, and W. Nolting, J. Phys.: Condens. Matter 19, 236213 (2007).

${ }^{33}$ J. Schliemann, J. König, and A. H. MacDonald, Phys. Rev. B 64, 165201 (2001).

${ }^{34}$ G. Zarand and B. Jankó, Phys. Rev. Lett. 89, 047201 (2002).

${ }^{35}$ L. Brey and G. Gómez-Santos, Phys. Rev. B 68, 115206 (2003).

${ }^{36}$ M. Berciu and R. N. Bhatt, Phys. Rev. B 69, 045202 (2004).

${ }^{37}$ M. Sawicki, K.-Y. Wang, K. W. Edmonds, R. P. Campion, C. R. Staddon, N. R. S. Farley, C. T. Foxon, E. Papis, E. Kamińska, A. Piotrowska, T. Dietl, and B. L. Gallagher, Phys. Rev. B 71, 121302(R) (2005); M. Sawicki, J. Magn. Magn. Mater. 300, 1 (2006).

${ }^{38}$ J. Schliemann and A. H. MacDonald, Phys. Rev. Lett. 88, 137201 (2002).

${ }^{39}$ H. B. Callen, Phys. Rev. 130, 890 (1963).

${ }^{40}$ J. Okabayashi, A. Kimura, O. Rader, T. Mizokawa, A. Fujimori, T. Hayashi, and M. Tanaka, Phys. Rev. B 58, R4211 (1998).

${ }^{41}$ F. Matsukura, H. Ohno, A. Shen, and Y. Sugawara, Phys. Rev. B 57, R2037 (1998).

${ }^{42}$ T. Omiya, F. Matsukura, T. Dietl, Y. Ohno, T. Sakon, M. Motokawa, and H. Ohno, Physica E (Amsterdam) 7, 976 (2000).

${ }^{43}$ S. Sanvito, P. Ordejón, and N. A. Hill, Phys. Rev. B 63, 165206 (2001).

${ }^{44}$ A. Singh, Phys. Rev. B 75, 035206 (2007). 